\title{
Energy-Saving Design for Hydraulic Tube Bender
}

\author{
J C Renn, C Y Cheng, and M H Lin, \\ Department of Mechanical Engineering, \\ National Yunlin University of Science and Technology, \\ Douliou, Yunlin, Taiwan \\ E-mail: rennjc@yuntech.edu.tw,g9511706@yuntech.edu.tw,m10011004@yuntech.edu.tw
}

\begin{abstract}
Generally speaking, hydraulic control systems can be divided into two different driving concepts. The first one is the wellknown valve-controlled system and the second one is the pump-controlled system. The former possesses the feature of fast dynamic response. However, the poor energy-saving performance is its major fault. On the contrary, the pump-controlled hydraulic system has the significant advantage of energy-saving which meets the current demand in modern machine design. In this paper, the simulation analysis using MatLab/SimuLink and DSHplus for a newly developed energy-saving hydraulic tube bender is conducted. Instead of the conventional fixed displacement hydraulic pump, the new hydraulic tube bender utilizes an internal gear pump with AC servomotor as its driving power source. In the new energy-saving hydraulic circuit, the use of conventional pressure relief valve and unloading valve are no longer necessary since the demanded flow-rate and pressure output can be precisely obtained by continuously changing the speed of the AC servomotor. In addition, two closed-loop control schemes using fuzzy sliding-mode controller are adopted and compared. From the simulation results, it is shown that the energy-saving performance of constant pressure control scheme is somewhat better than that of loadsensing control scheme. Furthermore, the simulation results also show that the newly developed hydraulic tube bender can save up to $42 \%$ of energy consumption in a working cycle as compared to the conventional hydraulic tube bender.
\end{abstract}

Keywords: Hydraulic Energy-Saving, Pump-Controlled System, Fluid Power, Tube Bender

\section{Introduction}

Nowadays, hydraulic systems still play a very important role in modern industry due to the large output force, high power/weight ratio and excellent stiffness. In this paper, a conventional hydraulic tube bender shown in Fig. 1 is chosen for the study of implementing the hydraulic energysaving design. Figure 2 shows a typical working cycle of the chosen hydraulic tube bender [5]. It is observed that eight sequential steps are required to complete the bending of a straight tube. They are: (A) advancing the main pushing unit, (B) clamping the tube, (C) advancing the bending unit together with auxiliary pushing unit and bending the tube (90 degrees), (D) retracting the main pushing unit, (E) releasing the clamping of tube, $(\mathrm{F})$ retracting the auxiliary pushing unit, $(\mathrm{G})$ removal of tube and $(\mathrm{H})$ retracting the bending unit. In the hydraulic circuit of a conventional tube bender, a fixed-displacement vane pump is generally utilized as shown in Fig. 3. In addition, a pilot-operated pressure balanced relief valve together with a two-way solenoid operated valve is used as well to construct the unloading circuit. However, the hydraulic supply pressure of the conventional tube bender has to be set at the maximal demanded pressure of 120 bar to ensure the proper function of all 8 steps. Thus, from Fig. 2, it is clear that huge energy lost in the working cycle is inevitable since only the step C requires the maximal supply pressure of 120 bar. For the rest 7 steps, however, the actually demanded supply pressure is much lower than the maximal pressure of 120 bar. On the other hand, recent performance improvements in the $\mathrm{AC}$ servomotor provide a promising alternative to design the energy-saving hydraulic circuit [2, 3, and 4]. In details, the demanded flow-rate and pressure output of the hydraulic pumping unit can be precisely obtained by continuously changing the speed of the AC servomotor. In this paper, therefore, the implementation of energy-saving design is mainly based on the AC servomotor-controlled scheme. In the following, the proposed energy-saving control scheme is firstly outlined.

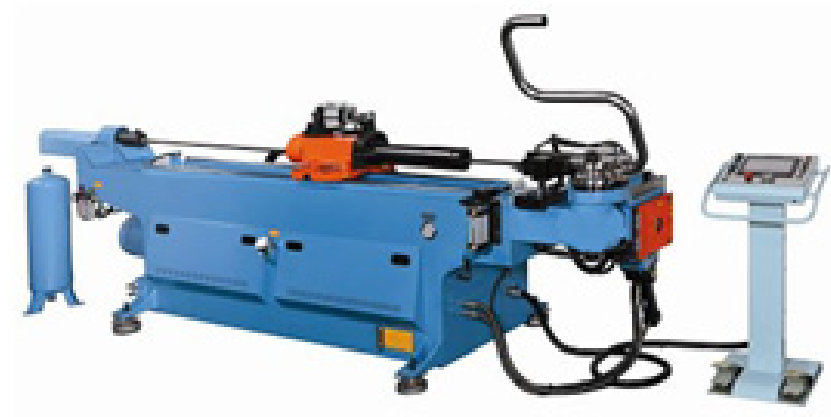

Figure 1: A hydraulic tube bender [5]. 


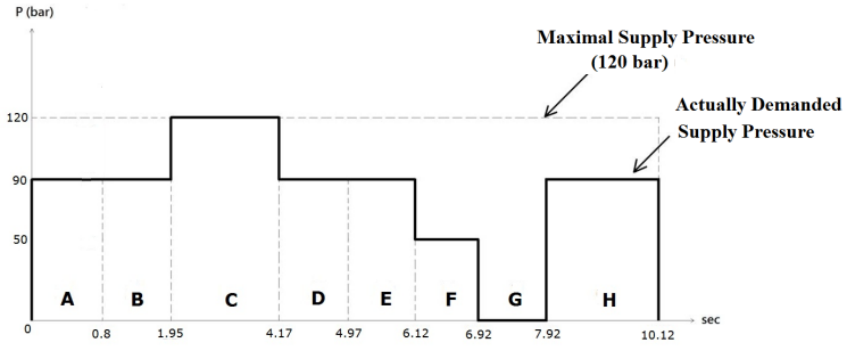

Figure 2: Demanded pressure in a working cycle.

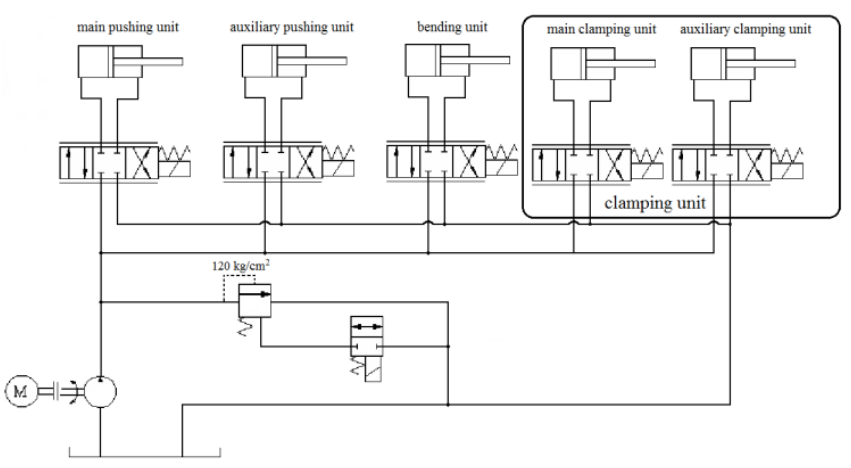

Figure 3: Schematic circuit diagram of a conventional hydraulic tube bender.

\section{Energy-saving control scheme}

In this paper, two closed-loop control schemes using fuzzy sliding-mode controller are adopted and compared. The first one is load-sensing control strategy and the other one is constant pressure control scheme [4]. Figure 4 shows the block diagram of the load-sensing control. A fixed pressure drop, $\Delta p_{\text {set }}$, across the control solenoid valve serves as the reference input. The load pressure, $p_{L s}$, is measured by a pressure sensor and fedback to the closed-loop control system to ensure that the supply pressure, $p_{s}$, can be realtime adjusted according to the variable load pressure. Consequently, the actual pressure drop, $\Delta p=p_{s}-p_{L s}$, can be maintained as a constant by the closed-loop control. On the other hand, Figure 5 shows the block diagram of constant pressure control. In this configuration, the reference supply pressure input, $P_{\text {sset }}$, is varied according to the different demanded pressure levels at various steps as shown in Fig. 2. Thus, the reference supply pressure input is actually not a constant and has to be changed at different steps. Besides, it is worth mentioning that the fuzzy sliding-mode scheme is chosen as the closed-loop controller because it provides a dither-like chattering signal. Such a signal is proven to be effective to overcome the nonlinear friction disturbances [1].

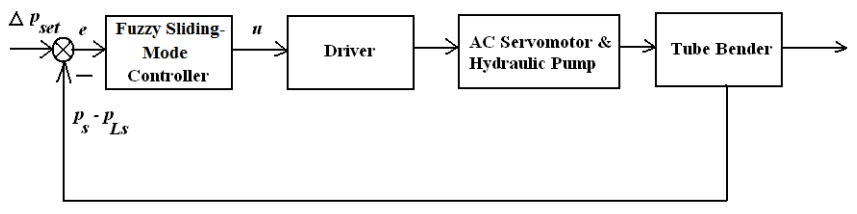

Figure 4: Load-sensing control block diagram.

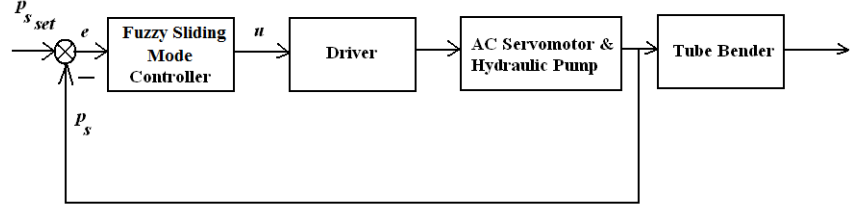

Figure 5: Constant pressure control block diagram.

The combination of fuzzy-logic-controller with sliding mode controller results in the fuzzy sliding-mode controller. As an example, the constant pressure control scheme combining the fuzzy sliding-mode controller is illustrated as follows. Figure 6 shows the schematic block diagram of the fuzzy sliding-mode controller. The error signal, $e(k)$, and the error signal change, $\Delta e(k)$, are denoted by $x_{1}$ and $x_{2}$ respectively, that is

$$
\begin{aligned}
& x_{1}=e(k)=p_{\text {sset }}-p_{s}, \\
& x_{2}=\Delta e(k)=e(k)-e(k-1),
\end{aligned}
$$

where $p_{\text {sset }}$ : reference supply pressure input,

$$
p_{s}: \text { actual supply pressure. }
$$

Unlike the conventional controller, there are four procedures involved in the implementation of a fuzzy sliding-mode controller, fuzzification of the input, fuzzy inference based on the knowledge, the defuzzification of the rule-based control signal and the determination of the scaling factors.

\subsection{Fuzzification}

The proposed fuzzy sliding-mode controller requires only one input signal, $s$, which is exactly the combination of the parameters $x_{1}$ and $x_{2}$.

$$
s=\lambda x_{1}+x_{2}
$$

Figure 7 shows the usual triangular fuzzy membership function for the input signal, $s$, and for the output signal, $u$. The input signal also represents the switching surface of the sliding mode controller.

\subsection{Inference}

The inference process consists of nine typical rules driven by the linguistic values of the input signal, $s$. There are:

If $S$ is very large-positive, then $u$ is very largepositive.

If $S$ is large-positive, then $u$ is large-positive. 
If $S$ is medium-positive, then $u$ is medium-positive.

If $s$ is small-positive, then $u$ is small-positive.

If $s$ is zero, then $u$ is zero.

If $s$ is small-negative, then $u$ is small-negative.

If $s$ is medium-negative, then $u$ is medium-negative.

If $s$ is large-negative, then $u$ is large-negative.

If $s$ is very large-negative, then $u$ is very largenegative.

\subsection{Defuzzification}

The defuzzification is to transform control signal into exact control output. In the defuzzification, the method of center of gravity is used.

$$
u=\frac{\sum_{i=1}^{n} W_{i} B_{i}}{\sum_{i=1}^{n} W_{i}},
$$

where $u$ : output of the fuzzy sliding-mode controller,

$$
\begin{aligned}
& W_{i}: \text { the degree of firing of the } i_{t h} \text { rule, } \\
& B_{i}: \text { the centroid of the consequent fuzzy subset } \\
& \text { of the } i_{t h} \text { rule. }
\end{aligned}
$$

\subsection{Determination of scaling factors, $G S$ and $G U$}

Before the input signal, $\mathrm{s}$, is fed into the fuzzy-sliding mode controller, it has to be multiplied by the scaling factor, $G S$, so that the product is normalized in the interval $[-1,1]$. Similarly, the output signal of the fuzzy-sliding mode controller, $u$, is multiplied by the scaling factor, $G U$, to meet the actual operating voltage range, $-10 \mathrm{~V}$ to $10 \mathrm{~V}$, of the $\mathrm{AC}$ servomotor. It is also worth mentioning that the values of the factors, $G S$ and $G U$, affect the system response greatly.

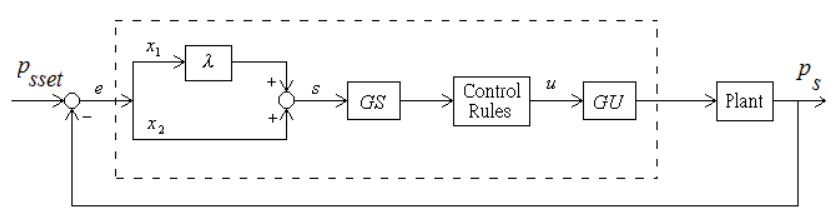

Figure 6: Scheme of the proposed fuzzy sliding-mode control.

\section{Modeling, Simulation results and Discussion}

In this paper, two commercial software packages, the MatLab/SimuLink and DSHplus, are chosen as simulation tools to analyze the energy-saving performance. Firstly, the modeling procedure by MatLab/SimuLink will be outlined.
The dynamics of the utilized AC servomotor can be simplified as a first-order function. In addition, a simplified model for a typical single-acting hydraulic cylinder is shown in Fig. 8. The volumetric flow-rate output of the hydraulic pump is given by [2]

$$
Q_{s}=V p \times\left(1-e^{-t / T}\right) N \text {, }
$$

where $V p:$ displacement of pump,

$$
\begin{aligned}
& T \text { : time constant of AC servomotor, } \\
& N \text { : reference rotational speed input. }
\end{aligned}
$$

In practice, the time constant, $T$, can be derived from the data sheet of the AC servomotor and is estimated to be 50 ms [6].

From continuity equation, the dynamic model for the supply pressure can be simplified as

$$
\frac{d P_{s}}{d t}=\frac{E_{\text {oil }}}{V_{\text {pipe }}}\left(Q_{s}-Q_{a}\right),
$$

where $Q_{a}$ denotes the volumetric flow-rate going into the chamber A of cylinder and can be described by

$$
Q_{a}=B X_{v} \sqrt{\left(P_{s}-P_{a}\right)}
$$

Similarly, the volumetric flow-rate coming out of the chamber B of cylinder can be obtained as

$$
Q_{b}=B X_{v} \sqrt{P_{b}}
$$

where $X_{v}$ : spool displacement,

$$
B \text { : flow constant. }
$$

Since the switching directional control valve is utilized in the circuit design, the spool displacement, $X_{v}$, is set to be the maximal stroke +3 or $-3 \mathrm{~mm}$.

The flow constant, $B$, in Eq. (8) can further be expressed as

$$
B=C_{d} \pi d_{v} \sqrt{2 / \rho},
$$

where $C_{d}$ : flow coefficient,

$$
\begin{gathered}
d_{v}: \text { diameter of the valve spool, } \\
\rho: \text { density of the hydraulic oil. }
\end{gathered}
$$


If the internal and external leakages of the cylinder are both negligible, the simplified dynamic pressure equations for both chambers of the cylinder are

$$
\begin{aligned}
& \frac{d P_{a}}{d t}=\frac{E_{\text {oil }}}{V_{a}+V_{\text {pipe }}}\left(Q_{a}-A \dot{Y}\right), \\
& \frac{d P_{b}}{d t}=\frac{E_{\text {oil }}}{V_{b}+V_{\text {pipe }}}\left(-Q_{b}+A \dot{Y}\right),
\end{aligned}
$$

where $E_{\text {oil }}$ : bulk modulus of oil,

$$
\begin{aligned}
& V_{a}: \text { volume of chamber } \mathrm{A}, \\
& V_{b}: \text { volume of chamber } \mathrm{B}, \\
& V_{\text {pipe }}: \text { volume of connecting pipe, } \\
& A: \text { average area of piston, } \\
& \dot{Y}: \text { velocity of the piston. }
\end{aligned}
$$

Finally, the equation of motion gives

$$
M \ddot{Y}+b \dot{Y}=A_{a} P_{a}-A_{b} P_{b}-F_{L},
$$

Where $M:$ mass of load,

$$
\begin{aligned}
& \ddot{Y}: \text { acceleration of piston, } \\
& \mathrm{b}: \text { Newton's friction coefficient, } \\
& A_{a}: \text { actuating area of piston in chamber } \mathrm{A}, \\
& A_{b}: \text { actuating area of piston in chamber } \mathrm{B}, \\
& F_{L}: \text { disturbance load. }
\end{aligned}
$$

Thus, the MatLab/SimuLink model for the single-acting hydraulic cylinder is shown in Fig. 8. Since there are totally five hydraulic cylinders in the conventional tube bender, the whole hydraulic circuit model containing five single-acting hydraulic cylinders built by MatLab/SimuLink is shown in Fig. 9. On the other hand, to double check the validity of the above mentioned modeling and simulation, the hydraulic circuit model using the second software package DSHplus for the same conventional tube bender is established as shown in Fig. 10. Figure 11 shows the simulation displacements of the main pushing cylinder by two different software packages respectively. Similarly, two calculated actuating pressure curves of the main pushing cylinder are also depicted in Fig. 12. Obviously, from Figures 11 and 12, the calculated cylinder displacements as well as actuating pressure curves derived from MatLab/SimuLink and DSHplus respectively are almost identical. It can therefore be verified that the modeling procedure and simulation results by MatLab/SimuLink are reliable and can be used for further energy-saving analysis.

It is worth mentioning that the block diagram shown in Fig. 9 is actually an open loop control scheme. It is necessary to incorporate the proposed fuzzy sliding-mode controller into the control structure and form a closed-loop control block diagram. In addition, two proposed control schemes, the constant pressure control and load-sensing control, have also to be implemented. Thus, based on the simplified block diagrams shown in Figures 4 and 5, two comprehensive closed-loop control block diagrams can be obtained as shown in Figures 13 and 14 respectively. In the following, the control performances of these two closed-loop control schemes will be discussed and compared.

After adequate simulations, all important data and information of the whole tube bender system, like the pressure and flow-rate etc., can be obtained. The power consumption of the pumping unit can easily be calculated by the following equation (13).

$$
P_{\text {pump }}=\frac{p_{s} \times Q_{s}}{600}
$$

where $P_{\text {pump }}$ : power consumption of the pump,

$$
\begin{aligned}
& p_{s}: \text { supply pressure, } \\
& Q_{s}: \text { supply flow-rate. }
\end{aligned}
$$

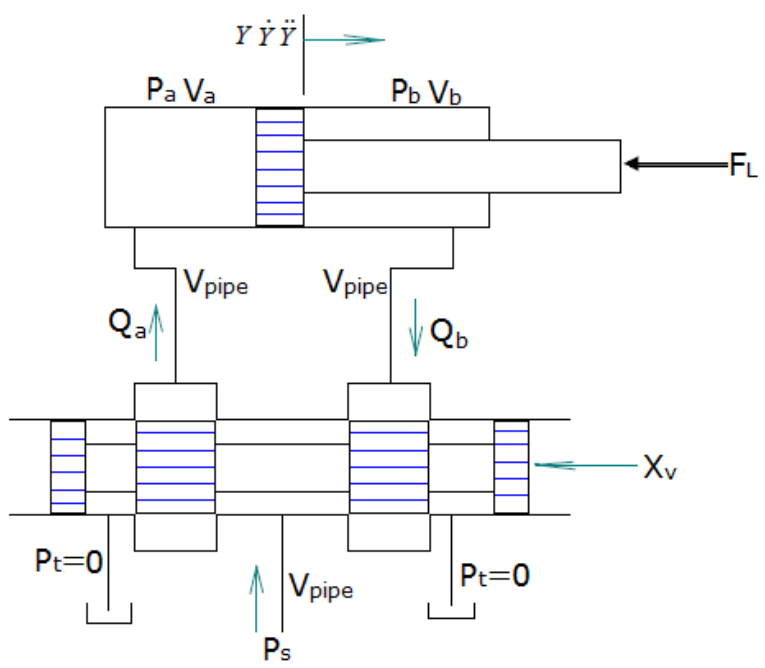

Figure 7: A simplified model for a single-acting hydraulic cylinder [2]. 


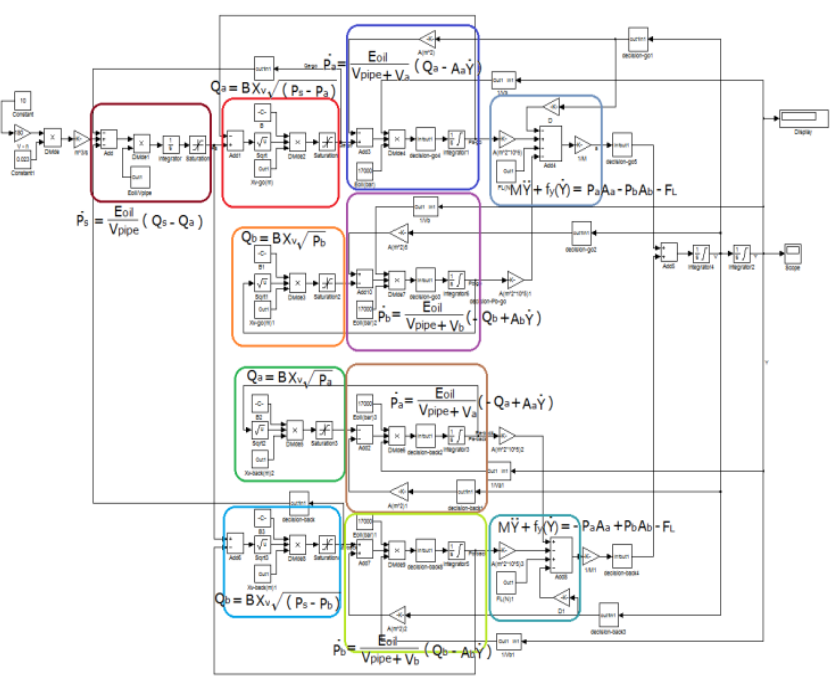

Figure 8: MatLab/SimuLink model for the single-acting hydraulic cylinder.

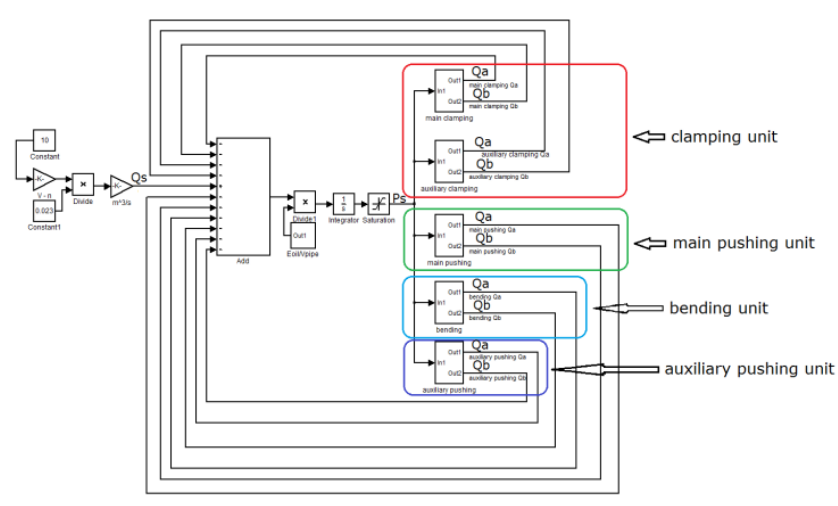

Figure 9: MatLab/SimuLink model for the conventional hydraulic tube bender.

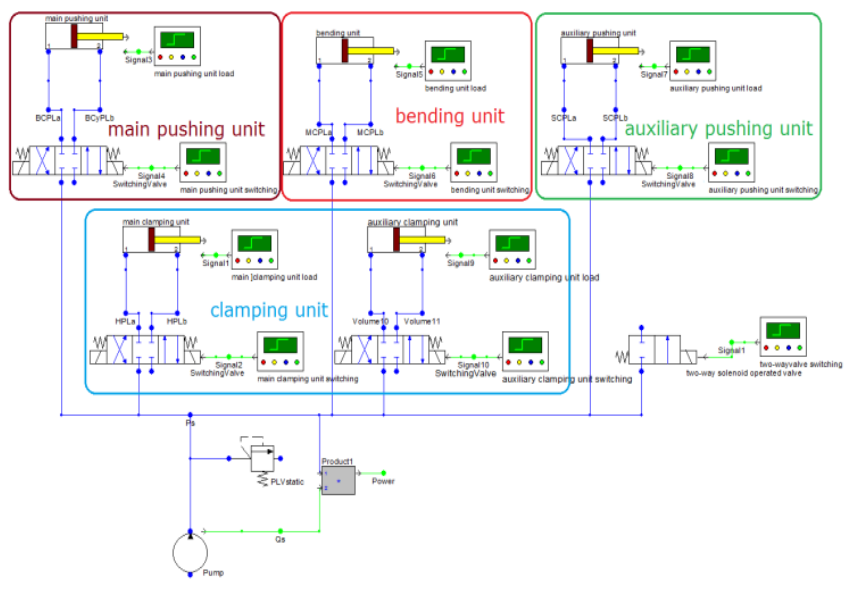

Figure 10: Hydraulic circuit model built by DSHplus for conventional tube bender.

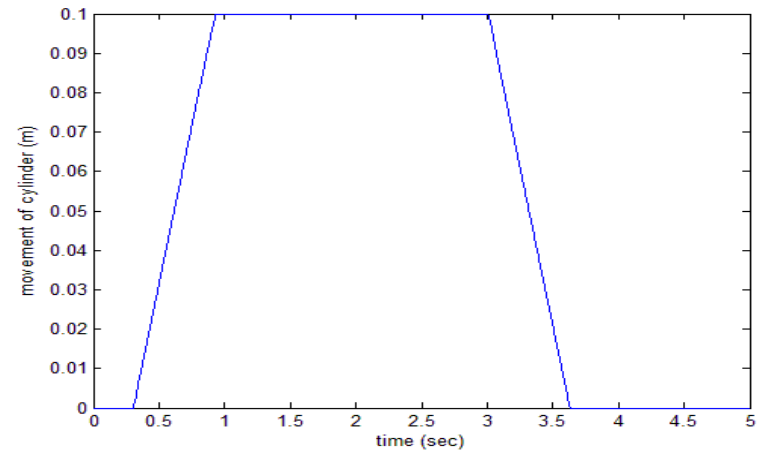

(a)

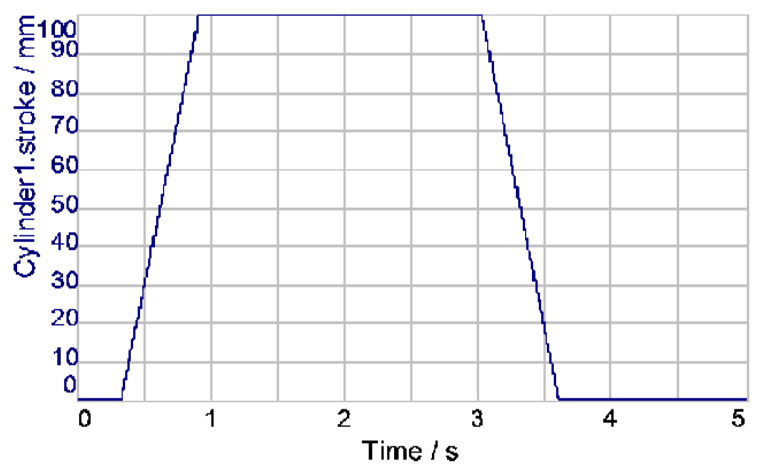

(b)

Figure 11: Simulation cylinder displacements of the main pushing unit by two different software packages (open loop).

(a) MatLab/SimuLink results; (b) DSHplus results

As an illustrated example, the simulation results of supplied actuating pressure and volumetric flow-rate by load-sensing control for the main pushing cylinder are shown in Fig. 15, where the pressure drop, $\Delta p_{\text {set }}$, is set to be 3 bar. It is noticeable that both the pressure and flow-rate curves for the advance and retraction of the main pushing cylinder are depicted. In addition, Figure 16 shows the similar simulation results by constant pressure control scheme, where the reference supply pressure input, $P_{\text {sset }}$, is set to be 90 bar according to the demanded pressure level at step A shown in Fig. 2. Comparing Fig. 15 and Fig. 16, it is actually not easy to tell the differences of the pressure and volumetric flowrate signals during the advance and retraction phases of the main pushing cylinder. However, if Eq. (13) and appropriate numerical calculations are applied to evaluate the power consumption, the difference of driving power between two utilized closed-loop control strategies for the main pushing cylinder in one working cycle is more obvious as shown in Fig. 17.

It is worth mentioning that the energy-saving analysis using DSHplus is not executed in this paper because the proposed fuzzy sliding-mode controller is not available in the current library of the commercial software package DSHplus. 


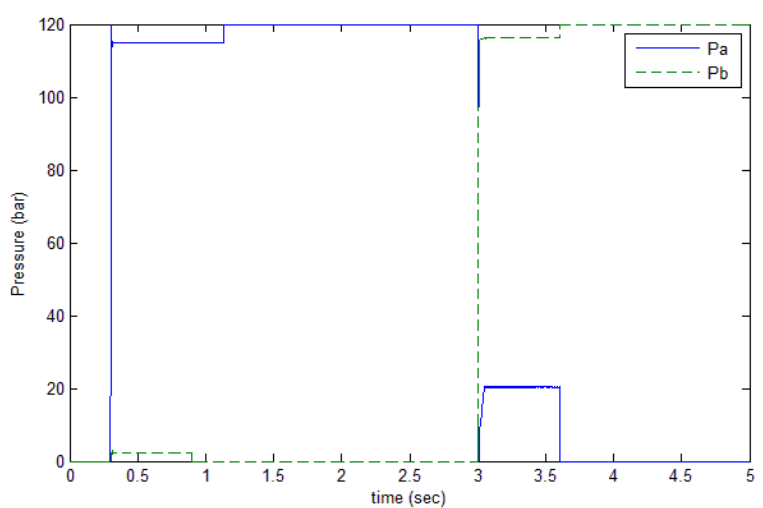

(a)

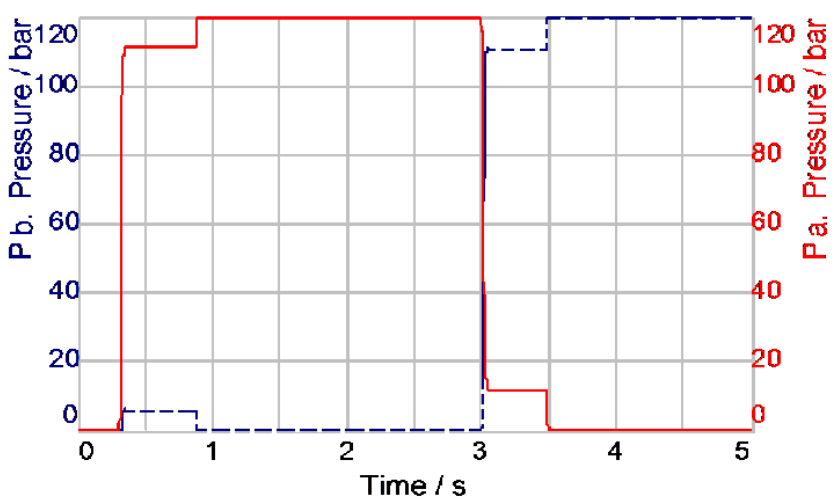

(b)

Figure 12: Simulation actuating pressure curves of the main pushing unit by two different software packages (open loop.)

(a) MatLab/SimuLink results; (b) DSHplus results

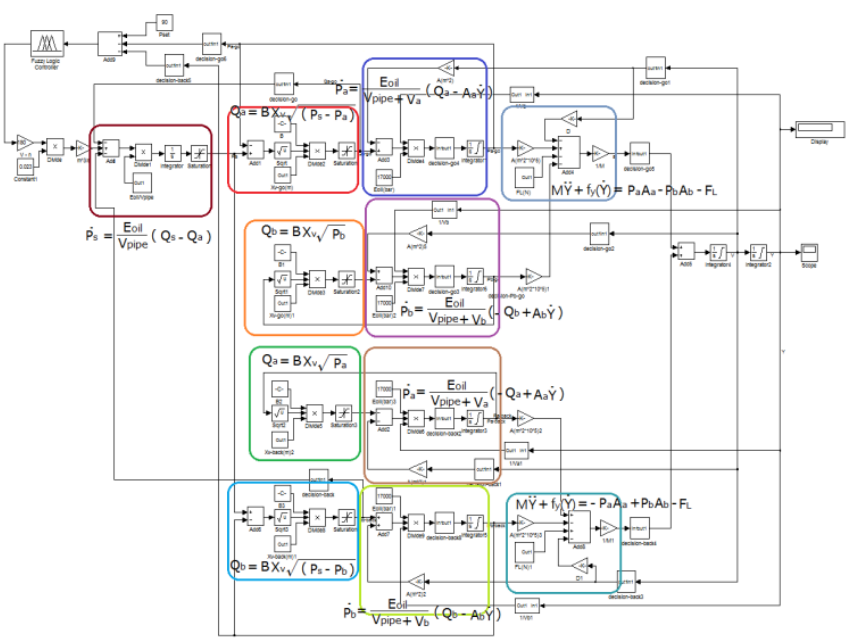

Figure 13: Closed-loop load-sensing control block diagram for the main pushing cylinder.

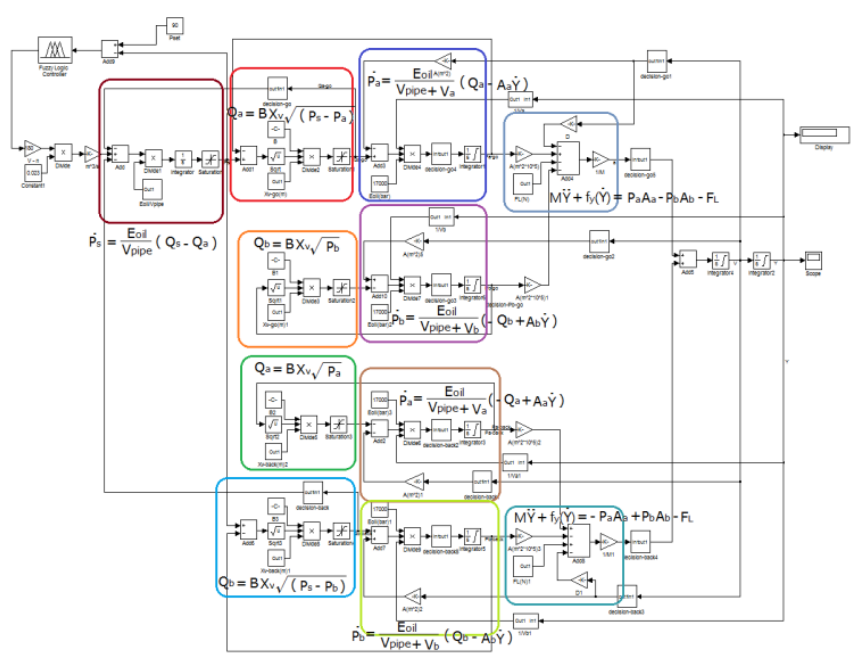

Figure 14: Closed-loop constant pressure control block diagram for the main pushing cylinder.

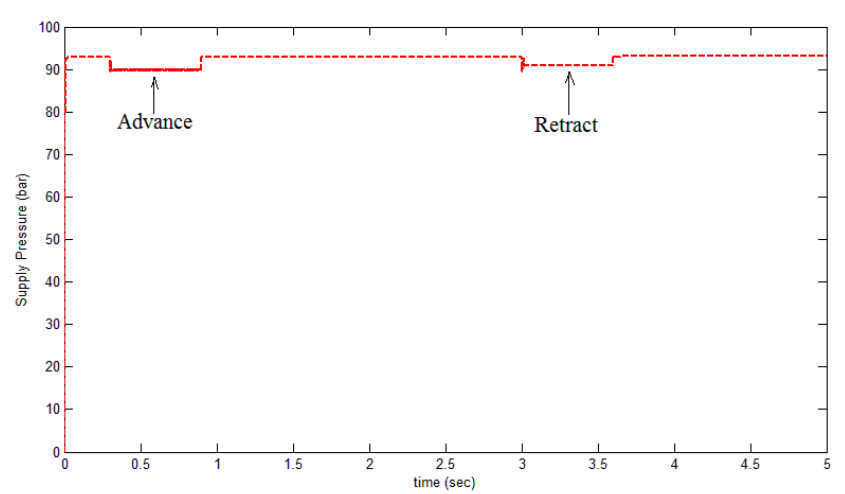

(a)

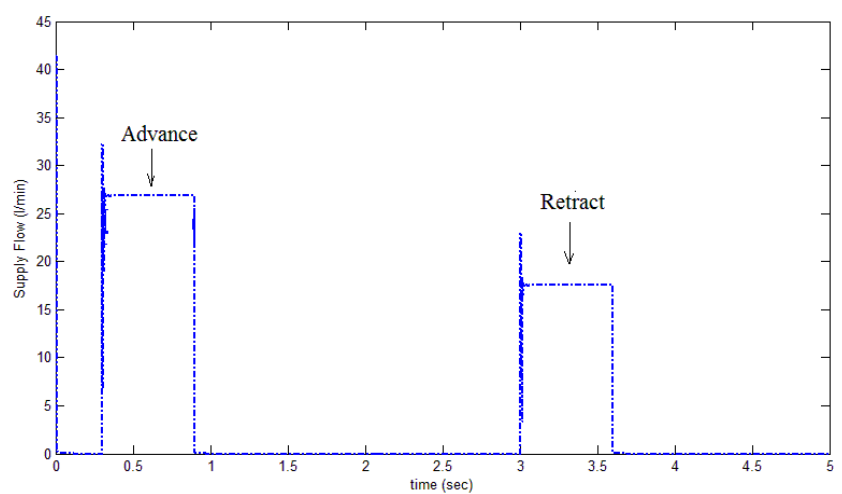

(b)

Figure 15: Simulation results of (a) supplied actuating pressure and (b) volumetric flow-rate by load-sensing control for the main pushing cylinder. 


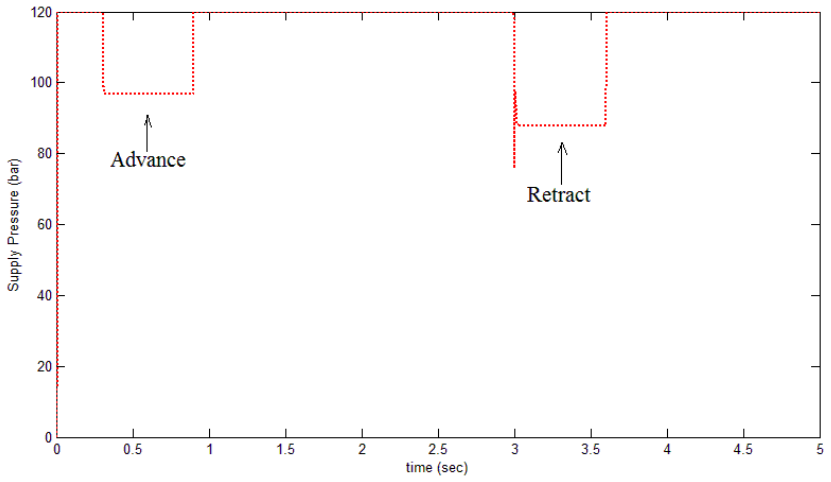

(a)

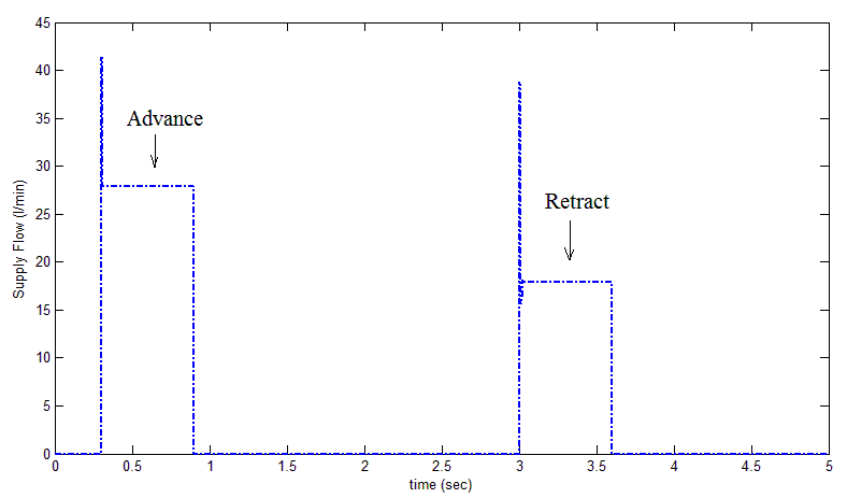

(b)

Figure 16: Simulation results of (a) supplied actuating pressure and (b) volumetric flow-rate by constant pressure control for the main pushing cylinder.

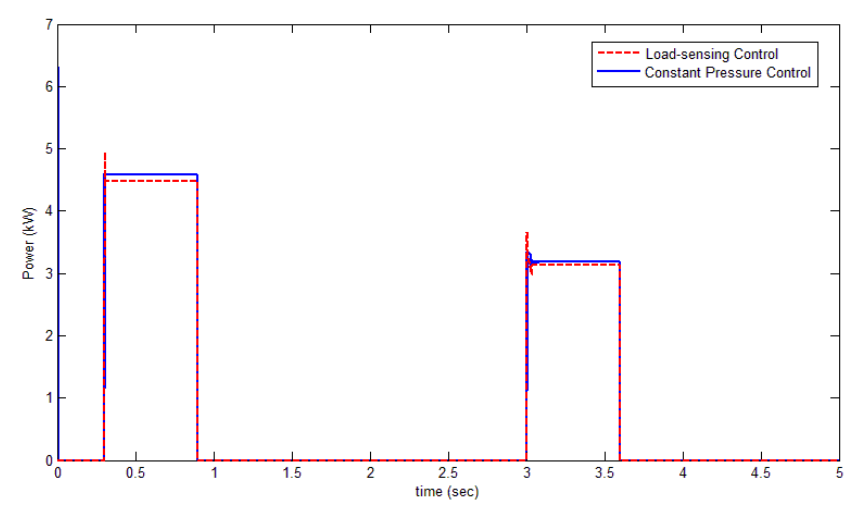

Figure 17: Simulation results of power consumption between two utilized closed-loop control strategies for the main pushing cylinder.

Table 1 shows the comparisons of overall energy consumption of pumping unit between the conventional and newly designed energy-saving hydraulic tube bender. It is observed that the energy-saving performance of constant pressure control scheme is somewhat better than that of load-sensing control scheme. Furthermore, the simulation results also show that the newly designed hydraulic tube bender can save up to $42 \%$ of energy consumption in a working cycle as compared to the conventional hydraulic tube bender. These results prove the validity of the proposed energy-saving hydraulic circuit design as well as the effectiveness of proposed closed-loop control schemes.

Table 1: Comparisons of energy consumption between conventional and new energy-saving hydraulic tube bender

\begin{tabular}{|c|c|c|c|c|c|c|c|c|c|}
\hline \multirow{2}{*}{ Step } & \multicolumn{3}{|c|}{ Conventional } & \multicolumn{3}{|c|}{ Load-sensing control } & \multicolumn{3}{|c|}{ Const. pressure control } \\
\hline & $P_{5}$ (bar) & $\mathrm{Qs}_{(1 / \mathrm{min})}$ & $\mathbf{P}_{\text {pump }}(\mathrm{kW})$ & $P_{s}$ (bar) & $\mathrm{Qs}_{(\mathrm{s}}(1 \mathrm{~min})$ & $\mathbf{P}_{\mathrm{Pmpm}}(\mathrm{kW})$ & $P_{\mathrm{s}}$ (bar) & $\mathrm{Q}_{(}(1 / \mathrm{min})$ & $\mathbf{P}_{\mathrm{pump}}(\mathrm{kW})$ \\
\hline A & 120 & 41.4 & 8.28 & 89.36 & 31.41 & 4.68 & 89.6 . & 32.58 & 4.86 \\
\hline B & 120 & 41.4 & 8.28 & \begin{tabular}{|l|}
89.97 \\
\end{tabular} & 33.08 & 4.96 & 89.6 & 32.43 & 4.84 \\
\hline C & 120 & 41.4 & 8.28 & 119,04 & 38.04 & 7.55 & 119.54 & 38.37 & 7.64 \\
\hline D & 120 & 41.4 & 8.28 & 89.96 & 22.06 & 3.31 & 89.71 & 23.93 & 3.58 \\
\hline E & 120 & 41.4 & 8.28 & 89.84 & 26.26 & 3.93 & 89.59 & 23.23 & 3.47 \\
\hline F & 120 & 41.4 & 8.28 & 51.04 & 17.42 & 1.48 & 49.82 & 14.38 & 1.19 \\
\hline G & 0 & 41.4 & 0 & 0 & 0 & 0 & 0 & 0 & 0 \\
\hline H & 120 & 41.4 & 8.28 & 89.94 & 27.51 & 4.12 & 89.6 & 28.2 & 4.21 \\
\hline $\begin{array}{l}\text { Total } \\
\text { Energy(kJ) }\end{array}$ & \multicolumn{3}{|c|}{75.51} & \multicolumn{3}{|c|}{43.62} & \multicolumn{3}{|c|}{43.46} \\
\hline $\begin{array}{l}\text { Energy- } \\
\text { Saving } \\
\text { Percentage }\end{array}$ & \multicolumn{3}{|c|}{$0 \%$} & \multicolumn{3}{|c|}{$42.23 \%$} & \multicolumn{3}{|c|}{$42.44 \%$} \\
\hline
\end{tabular}

\section{Conclusions}

In this paper, a new energy-saving hydraulic tube bender is successfully designed based on the simulation analysis using MatLab/SimuLink and DSHplus. The introduction of the additional commercial software package DSHplus is merely to verify the validity of proposed modeling procedure and simulation results. In addition, three conclusions may also be drawn from this research.

(1) The simulation results derived from MatLab/SimuLink and DSHplus respectively are nearly identical. It can therefore be verified that the modeling procedure and simulation results by MatLab/SimuLink are reliable and can be used for further energy-saving analysis.

(2) Simulation results show that the newly designed hydraulic tube bender can save up to $42 \%$ of energy consumption in a working cycle as compared to the conventional hydraulic tube bender. This result not only shows the potential of energy-saving design but also encourages the future real implementation of the new tube bender.

(3) The energy-saving performance of constant pressure control scheme is somewhat better than that of loadsensing control scheme. Besides, the former utilized only one pressure sensor while the latter requires two pressure sensors to calculate the actual pressure drop. Therefore, the better choice would be undoubtedly the constant pressure control scheme which will be suggested to be implemented in the future real layout of the new hydraulic tube bender. 


\section{Acknowledgement}

The financial support of the National Science Council under grant number NSC-101-2622-E-224-014-CC3 is greatly appreciated. In addition, the industrial project supported by SOCO Machinery Co., LTD. is also acknowledged.

\section{Nomenclature}

\begin{tabular}{|c|c|c|}
\hline Designation & Denotation & Unit \\
\hline$A$ & Piston Average Area & {$\left[\mathrm{m}^{2}\right]$} \\
\hline$A_{a}$ & $\begin{array}{l}\text { Piston Actuating Area (Chamber } \\
\text { A) }\end{array}$ & {$\left[\mathrm{m}^{2}\right]$} \\
\hline$A_{b}$ & $\begin{array}{l}\text { Piston Actuating Area (Chamber } \\
\text { B) }\end{array}$ & {$\left[\mathrm{m}^{2}\right]$} \\
\hline$B$ & Flow Constant & $\begin{array}{l}{\left[\mathrm{m}^{2} / \mathrm{min}\right.} \\
\sqrt{\text { bar }]}\end{array}$ \\
\hline $\mathrm{b}$ & Newton's Friction Coefficient & {$[\mathrm{N} \mathrm{s} / \mathrm{m}]$} \\
\hline$C_{d}$ & Flow Coefficient & {$[-]$} \\
\hline$d_{v}$ & Spool Diameter & {$[\mathrm{mm}]$} \\
\hline$E_{\text {oil }}$ & Oil Bulk Modulus & [bar] \\
\hline$F_{L}$ & Disturbance Load & {$[\mathrm{N}]$} \\
\hline$M$ & Mass of Load & {$[\mathrm{kg}]$} \\
\hline$N$ & Reference Rotational Speed Input & {$[\mathrm{rpm}]$} \\
\hline$p_{L s}$ & Load-sensing Pressure & [bar] \\
\hline$p_{s}$ & Supply Pressure & [bar] \\
\hline$Q_{a}$ & $\begin{array}{l}\text { Volumetric Flow-rate going into } \\
\text { the Chamber A }\end{array}$ & {$[1 / \mathrm{min}]$} \\
\hline$Q_{b}$ & $\begin{array}{l}\text { Volumetric Flow-rate coming out } \\
\text { of the Chamber B }\end{array}$ & {$[1 / \mathrm{min}]$} \\
\hline$Q_{s}$ & Supply Flow-rate & {$[1 / \mathrm{min}]$} \\
\hline$\Delta p_{\text {set }}$ & Reference Pressure Drop Input & [bar] \\
\hline$P_{\text {sset }}$ & Reference Supply Pressure Input & [bar] \\
\hline $\boldsymbol{P}_{\text {pump }}$ & Power Consumption of Pump & {$[\mathrm{kW}]$} \\
\hline$T$ & time constant of AC servomotor & {$[\mathrm{s}]$} \\
\hline$u$ & Actuating Signal & {$[$ Volt $]$} \\
\hline$V p$ & Pump Displacement & {$[\mathrm{cc}]$} \\
\hline$V_{a}$ & Volume of Chamber A & {$\left[\mathrm{m}^{3}\right]$} \\
\hline$V_{b}$ & Volume of Chamber B & {$\left[\mathrm{m}^{3}\right]$} \\
\hline$V_{\text {pipe }}$ & Volume of Connecting Pipe & {$\left[\mathrm{m}^{3}\right]$} \\
\hline$X_{v}$ & Spool Displacement & {$[\mathrm{mm}]$} \\
\hline$Y$ & Displacement of Cylinder Piston & {$[\mathrm{m}]$} \\
\hline$\dot{Y}$ & Velocity of Cylinder Piston & {$[\mathrm{m} / \mathrm{s}]$} \\
\hline
\end{tabular}

\section{References}

[1] J C Renn, Position Control of A Pneumatic Servocylinder Using Fuzzy-sliding Surface Controller, Int. J. of Fluid Power, 3(3): 19-25, 2002.

[2] H E Merritt, Hydraulic Control Systems, John Wileys and Sons, Cincinnati, Ohio, 1967. ISBN 0-471-59617-5.

[3] H Murrenhoff, Systematic approach to the control of hydrostatic drives, Proc. of the Institution of Mechanical Engineers. Part I: Journal of Systems \& Control Engineering, 213(I5): 333-347, 1999.

[4] M H Chiang, Y W Chien and D D Yu, Implementation of integrated control of path control and energy-saving control for hydraulic servo systems," J CSME, 24(2): 50-58, 2003.

[5] N N, Hydraulic Tube Bender, Catalogue of SOCO machinery Co., LTD, www.soco.com.tw, 2012.

[6] N N, HES Series AC Servomotor, Catalogue of Delta Electronics, Inc., http://www.delta.com.tw, 2012. 